\title{
La réfractivité radar : vers une cartographie de l'humidité en très basse couche de l'atmosphère
}

\author{
Lucas Besson ${ }^{(1)}$, Olivier Caumont ${ }^{(2)}$, Jacques Parent du Châtelet ${ }^{(3)}$ \\ (1) Laboratoire atmosphères, milieux, observations spatiales (Latmos), \\ Institut Pierre-Simon Laplace (IPSL), UPMC, 4 place Jussieu, \\ 75005 Paris \\ (2) CNRM-Game, Météo-France et CNRS, 42 avenue Coriolis, \\ 31057 Toulouse Cedex 1 \\ (3) DSO/CMI Météo-France et Latmos, 11 boulevard d'Alembert, \\ 78280 Guyancourt
}

\section{Résumé}

Les radars météorologiques ont été initialement conçus pour détecter et quantifier les précipitations. Une nouvelle technique a été proposée à la fin des années 1990 pour mesurer le changement de l'indice de réfraction de l'air dans les basses couches de l'atmosphère en exploitant la phase du signal provenant de cibles fixes situées autour du radar. Cette mesure donne des informations sur l'indice de réfraction de l'air, lui-même combinaison de la pression, de la température et de l'humidité, et présente donc un intérêt météorologique, aussi bien pour la prévision numérique que pour les études de processus.

\section{Abstract}

Radar refractivity: towards mapping the moisture content of the lowest part of the atmosphere

Weather radars were originally conceived to detect and quantify precipitation. In the late 90s a new technique was proposed to measure the change in the refractive index of air in the lower layers of the atmosphere through the use of the phase of the signal from ground targets located around the radar. This measure provides information on the refractive index of air (itself a combination of pressure, temperature, and moisture) and so is of meteorological interest, both for numerical weather prediction and process studies. es radars météorologiques sont des instruments essentiels pour la localisation et l'estimation des précipitations. Actuellement, ils représentent la pierre angulaire du système de surveillance et d'alerte des agences météorologiques qui s'appuient sur des réseaux radars opérationnels, permettant une observation volumique de l'atmosphère sur l'ensemble du territoire, avec une résolution spatiale de l'ordre du kilomètre. Cependant, l'occurrence des précipitations est faible par rapport aux périodes de ciel non précipitant et ces radars sont sous-employés en regard de leur potentiel.

À partir de ce constat, Fabry et al.(1997) ont proposé d'utiliser les échos radar provenant du sol pour mesurer l'indice de réfraction des basses couches de l'atmosphère, ce qui donne des informations sur les variations des paramètres météorologiques tels que la pression, la température et surtout l'humidité. Cette technique originale a été développée pour les radars en bande $\mathbf{S}(\lambda=10 \mathrm{~cm})$ cohérents équipés d'émetteurs à klystron. La particularité de cette technologie réside dans la très grande stabilité de la fréquence d'émission, essentielle pour une estimation fiable et précise des fluctuations de l'indice de réfraction atmosphérique.

La réfractivité est d'autant plus sensible à l'humidité que la température est élevée. Ainsi, à $20^{\circ} \mathrm{C}$, une variation d'une unité de réfractivité correspond à une variation de $1 \%$ d'humidité relative. En été, les variations de réfractivité sont principalement dues à des changements d'humidité et le champ de réfractivité peut être utilisé pour estimer le champ d'humidité dans les très basses couches
(Weckwerth et al., 2005 ; Roberts et al., 2008). Les variations d'humidité de petite échelle, accessibles par le biais de la réfractivité par radar, présentent un intérêt météorologique certain, en particulier pour l'étude de l'initiation de la convection (Weckwerth et al., 2005 ; Demoz et al., 2006 ; Fabry, 2006 ; Wakimoto et Murphey, 2010 ; Bodine et al., 2010). En particulier, des études (Montmerle et al., 2002 ; Sun, 2005) ont souligné que la réfractivité radar pouvait être intéressante pour l'assimilation de données dans les modèles numériques de prévision du temps. Enfin, Heinselman et al. (2009) ont montré que les champs de réfractivité constituent une information complémentaire pour les prévisionnistes, utile pour analyser l'environnement proche de la surface et améliorer leur connaissance des masses d'air humide.

À partir d'une simulation Méso-NH d'un cas convectif typique du sud de la France, Besson et al. (2012) ont pu identifier la présence d'une signature en réfractivité particulière à l'avant de la convection, résultat de l'effet du courant de densité généré par les précipitations. L'intérêt est évident pour localiser les systèmes convectifs et prévoir leurs évolutions. Plus généralement, cette mesure a le potentiel de fournir des cartes de masse d'air en très basses couches à l'échelle kilométrique, jusqu'ici jamais encore observées, et qui amélioreront sans nul doute la connaissance des processus atmosphériques.

Cette nouvelle technique se place dans un contexte très évolutif pour la mesure de l'humidité atmosphérique : en dehors des réseaux d'observation au sol classiques ou des sondes embarquées sur des ballons, plusieurs systèmes 
sont actuellement à l'étude pour compléter les réseaux opérationnels : - les mesures in situ par des sondes embarquées sur des avions de ligne, mais les profils resteront limités au voisinage des aéroports ;

- certaines études (Gossard et al., 1998 ; Stankov et al., 2003) ont mis en évidence une relation entre les changements locaux de l'indice de réfraction et le paramètre de structure de la turbulence mesuré par les radars profileurs de vent, mais aucune application opérationnelle n'est encore envisagée ;

- l'humidité atmosphérique est aussi estimée indirectement à l'aide du radiomètre multicanal micro-onde, instrument passif, qui mesure les températures de brillance (équivalentes aux luminances par la loi du corps noir) observées dans la raie d'absorption de la vapeur d'eau (22,235 GHz). La résolution spatiale de ce type d'instrument reste réduite, ce qui en limite l'intérêt dans le cadre d'une application opérationnelle ;

- une estimation de l'humidité atmosphérique peut aussi être obtenue, suite à une excitation par lidar, par le retour de la raie de diffusion Raman de la molécule d'eau. Mais cet effet est peu énergétique et son utilisation reste en général limitée aux mesures de nuit, non bruitées par la lumière du soleil. La technique du lidar Dial (Dabas et Flamant, 2002) semble plus prometteuse, mais elle reste pour le moment limitée aux applications de recherche, car la maîtrise de la longueur d'onde du laser, nécessaire pour se placer exactement dans la raie d'absorption de la vapeur d'eau, est très difficile ;

- l'exploitation des retards atmosphériques des signaux du réseau de satellites GPS est actuellement la technique la plus aboutie (Doerflinger, 2001). De manière analogue au radar dont il est question dans cet article, ce retard est fonction de l'indice de réfraction atmosphérique intégré le long du trajet entre le satellite et la station au sol. Les retards zénithaux audessus des stations GPS sont maintenant estimés en opérationnel et échangés au niveau européen pour être assimilés par certains modèles numériques de prévision du temps. Des efforts sont également en cours pour appliquer les méthodes de tomographie aux signaux de plusieurs satellites reçus par plusieurs stations au sol, de façon à restituer la répartition volumique de l'indice de réfraction avec une résolution horizontale essentiellement déterminée par l'espacement des stations au sol.

Comme la réfractivité, qu'elle soit obtenue par GPS ou par radar, dépend assez simplement des paramètres primaires du modèle de prévision du temps, ce dernier pourra l'utiliser directement à travers un schéma d'assimilation simple. À condition d'admettre un modèle pour la pression et la température, la réfractivité peut également être transformée en humidité atmosphérique pour des études de processus.

Parmi toutes les innovations évoquées plus haut, la technique de réfractivité par radar présente l'originalité de fournir des cartes de répartition de l'humidité dans un rayon de $50 \mathrm{~km}$ autour du radar avec une très bonne résolution, actuellement de l'ordre de quelques kilomètres, mais qui pourrait descendre à l'échelle hectométrique. Le fait que la mesure reste limitée aux très basses couches atmosphériques, puisque le radar et les cibles sont tous deux au niveau du sol, présente également un intérêt particulier pour comprendre les échanges entre le sol et l'atmosphère.

C'est avec ces motivations que MétéoFrance a décidé, en 2005, de travailler sur la mesure de réfractivité à partir des radars opérationnels, en commençant par le financement d'une bourse de thèse attribuée en commun par le Centre national de recherches météorologiques (CNRM) et la Direction des systèmes d'observation (DSO).

Cet article expose les étapes franchies depuis lors :

- adaptation du formalisme mathématique aux radars non cohérents (émetteur à magnétron) qui équipent le réseau Aramis et la plupart des réseaux opérationnels mondiaux;

- adaptation aux contraintes d'exploitation imposées par l'observation opérationnelle de la pluie ;

- comparaison à l'ébauche et à l'analyse du modèle de prévision du temps Arome, première étape à franchir avant l'assimilation ;

- validation en condition opérationnelle lors de la campagne HyMeX.

\section{Concept de la mesure de réfractivité}

L'indice de réfraction $(n)$ est le rapport entre les vitesses de propagation de l'onde électromagnétique dans le milieu traversé et dans le vide. Comme l'indice de l'air est très légèrement supérieur à 1, on définit par commodité la réfractivité $N$ par (voir par exemple Bean et Dutton, 1968) :

$$
N=(n-1) \times 10^{6}
$$

Aux longueurs d'onde centimétriques, Smith et Weintraub (1953) ont proposé une relation empirique entre la réfractivité et les variables météorologiques :

$$
N=77,6 \frac{P}{T}+3,73 \times 10^{5} \frac{e}{T^{2}}
$$

avec $P$ la pression (hPa), $T$ la température $(\mathrm{K})$ et $e$ la pression partielle de vapeur d'eau $(\mathrm{hPa})$.

Le concept de réfractivité radar repose sur la mesure du temps mis par une impulsion électromagnétique pour parcourir la distance aller-retour entre le radar et une cible fixe. La distance parcourue par l'impulsion étant constante, les fluctuations du temps de parcours sont liées aux changements d'indice de réfraction atmosphérique dus aux variations des paramètres atmosphériques le long du trajet.

Ces fluctuations du temps de parcours sont petites, de l'ordre de quelques nanosecondes. Elles n'ont pratiquement aucun effet sur l'instant de détection du signal, mais sont détectables par des changements de phase du signal reçu.

Fabry et al. (1997) ont établi que la variation temporelle de la phase de l'onde électromagnétique est reliée à la variation temporelle de la réfractivité, intégrée entre le radar et la cible de distance $r$, par:

$$
\Delta \phi=\frac{4 \pi f}{c} 10^{-6} \int_{0}^{\mathrm{r}} \Delta N(x) \mathrm{d} x
$$

$\Delta N$ est ici le changement de réfractivité entre les deux instants de mesure considérés et $f$ est la fréquence radar.

Pour accéder à une mesure de la réfractivité, l'un des deux instants de mesure est un temps de référence $t_{\text {ref }}$ pour lequel on suppose la réfractivité connue, égale à $N_{\text {ref. }}$ L'instant de mesure et l'instant de référence peuvent être séparés de plusieurs mois.

Pour accéder à une mesure locale et non pas intégrée, il faut effectuer une différentiation spatiale entre des cibles fixes situées sur la même radiale, à des distances voisines $r_{1}$ et $r_{2}$ entre lesquelles la réfractivité est supposée constante :

$$
\begin{gathered}
\mathrm{d} \phi\left(t, t_{\mathrm{ref}}, r\right)_{1 \rightarrow 2}=\frac{4 \pi f}{c} 10^{-6}\left[r_{2}-r_{1}\right] \\
{\left[N(r, t)-N\left(r, t_{\mathrm{ref}}\right]\right.}
\end{gathered}
$$

où $r$ est la distance moyenne égale à $\left(r_{1}+r_{2}\right) / 2$. 
Pour les radars non cohérents comme ceux qui constituent le réseau Aramis, la formulation est un peu plus complexe et certaines corrections doivent être apportées (voir l'encadré sur l'expression de la phase du signal pour un radar non cohérent).

\section{Ambiguïté de mesure et échos fixes}

\section{Repliement de phase}

L'estimation de la réfractivité radar s'effectue grâce à la mesure de la variation de la phase pendant un intervalle de temps $(\mathrm{d} t)$ et sur une distance $(\mathrm{d} r)$. La variation de phase ne peut être estimée correctement que lorsqu'elle est comprise dans l'intervalle $[-\pi ; \pi]$ pendant $\mathrm{d} t$ et sur $\mathrm{d} r$. Une ambiguiité de mesure est constatée lorsque la variation de phase sort de cet intervalle, impliquant que la variation de phase n'est plus connue qu'à $2 \pi$ près.

De nombreux facteurs peuvent être la cause de cette ambiguïté de mesure.

Tout d'abord, ces erreurs dépendent des caractéristiques du radar et des paramètres de la mesure : à partir d'une simulation utilisant des jeux de données indépendants, Besson et al. (2012) ont donné une formulation du taux d'ambiguïté en fonction de la fréquence d'émission du radar, de la distance d'intégration $\mathrm{d} r$ et de l'intervalle de temps entre deux échantillons successifs $\mathrm{d} t$.

Une seconde source d'erreur plus difficile à traiter trouve son origine dans la nature de la cible (végétation/immeuble, cibles isolées/multiples, contamination par des échos de pluie...). Suivant la nature de la cible, des fluctuations temporelles parfois difficiles à appréhender vont augmenter ou diminuer le temps de trajet de l'onde radar, et pourront se traduire par des ambiguïtés de mesure. Cette variation du temps de trajet peut être la conséquence de la présence d'eau liquide/solide sur le parcours de l'onde, mais aussi d'un faible déplacement de la cible (mouvement des arbres sous l'effet du vent) ou de l'effet de plusieurs cibles dans une même porte radar.

Pour améliorer l'estimation de la réfractivité radar, il est nécessaire :

- d'identifier les échos fixes et de sélectionner ceux qui sont aptes à la mesure de la réfractivité ;

\section{Expression de la phase du signal pour un radar non cohérent}

La formulation $\Phi=4 \pi f r n / c$ figure depuis longtemps dans les ouvrages de référence sur le radar, où elle constitue la base de nombreuses applications. Elle donne la phase d'un signal radar de fréquence $f$ après propagation aller-retour dans un milieu d'indice $n$ jusqu'à une cible située à la distance $r$.

L'application la plus classique est celle de la mesure de vitesse radiale de la cible par effet Doppler : si la distance $r$ varie linéairement en fonction du temps, en $\left[r(t)=v_{\text {radiale }}\right.$ $t+x_{0}$ ], le changement de phase entre deux instants de mesure séparés par $\Delta t$ sera donné par $\Delta \Phi=4 \pi f\left(v_{\text {radiale }}\right) \Delta t n / c$, ce qui permet de calculer simplement la vitesse radiale $v_{\text {radiale }}$ à partir de la mesure du changement de phase du signal $\Delta \Phi$.

Pour l'application un peu moins classique de la réfractivité, on utilise la même formulation pour relier le changement de phase $\Delta \Phi$ au changement d'indice de réfraction entre les deux instants de la mesure, la distance $r$ restant cette fois constante : $\Delta \Phi=4 \pi f r \Delta n / c$

Dans les deux applications évoquées ci-dessus, on suppose implicitement que les autres variables ( $f$ et $n$ pour le Doppler ; $f$ et $r$ pour la réfractivité) restent constantes pendant l'intervalle de temps qui sépare les deux mesures. Cette supposition semble raisonnable pour le Doppler avec des intervalles de temps de l'ordre de quelques centaines de millisecondes ; elle l'est beaucoup moins pour la réfractivité, avec des intervalles de temps de l'ordre de plusieurs heures, voire plusieurs jours.

La formulation de la phase d'un signal réfléchi par une cible distante a donc dû être revue pour prendre en compte les variations de la fréquence d'émission, particulièrement importantes pour les systèmes d'émission à magnétron, qui sont les plus répandus parce que les moins onéreux. Pour simplifier, un magnétron est une cavité placée dans un fort champ magnétique. Lorsqu'on applique un champ électrique, la cavité produit des oscillations électriques dont la fréquence est déterminée par la dimension de la cavité, elle-même légèrement variable en fonction de la température du milieu en raison de la dilatation. Pour les radars à précipitations classiques de longueur d'onde 5 ou $10 \mathrm{~cm}$, un changement de température de $1{ }^{\circ} \mathrm{C}$ se traduit par une variation de fréquence de l'ordre de $100 \mathrm{kHz}$.

En traitant la question de façon rigoureuse, Parent du Châtelet et al. (2012) ont montré qu'il faut prendre en compte dans le calcul non seulement la fréquence d'émission $f(t)$ du radar, mais aussi la fréquence $f_{\mathrm{LO}}(t)$ de l'oscillateur local par laquelle le signal reçu est multiplié pour être exploitable. C'est en effet sur ce dernier signal, après multiplication, que la mesure de phase est effectuée. Pour les systèmes radar dont la fréquence d'émission varie, comme les émetteurs à magnétron, $f_{\mathrm{LO}}(t)$ est en permanence automatiquement ajustée pour suivre les changements de la fréquence émise par le magnétron.

L'expression du changement de phase $\Delta \Phi$ entre deux instants $t_{\text {ref }}$ et $t$ du signal échantillonné au retard $\tau_{\text {sam }}$ est alors :

$$
\Delta \Phi\left(\tau_{\mathrm{sam},}, t, t_{\mathrm{ref}}\right)=2 \pi\left[\begin{array}{c}
-\left[f_{\mathrm{LO}}(t)-f_{\mathrm{LO}}\left(t_{\mathrm{ref}}\right)\right] \tau_{\mathrm{sam}} \\
+\left[f(t)-f\left(t_{\mathrm{ref}}\right)\right] \Delta \tau \\
-f\left(t_{\mathrm{ref}}\right) \frac{2 \times 10^{-6}}{c} f\left(t_{\mathrm{ref}}\right) \int \delta N\left(x, t_{\text {ref, }} t\right) \mathrm{d} x
\end{array}\right]
$$

Cette équation est la somme de trois termes :

- le terme d'oscillateur local qui est le produit du retard $\tau_{\text {sam }}$ par le changement de fréquence des oscillateurs locaux entre les instants $t_{\text {ref }}$ et $t$

- un terme " résiduel » qui est le produit du changement de fréquence d'émission par $\Delta \tau$ qui est la différence entre le vrai retard et le temps d'échantillonnage $\tau_{\text {sam }}$;

- un terme « de réfractivité » très semblable à la formulation classique de l'équation (3).

Le résultat le plus surprenant est que le changement de phase dépend en fait assez peu du changement de la fréquence d'émission $f(t)$ puisque $\Delta t$ reste assez petit ; par contre, il dépend beaucoup plus du changement de la fréquence de l'oscillateur local, ce qui se révèle finalement plutôt agréable, car cette fréquence est beaucoup plus facile à maîtriser que celle du signal émis.

Ce formalisme a été validé lors de diverses campagnes de tests sur différents radars de Météo-France (Parent du Châtelet et al., 2012). 
- et de diminuer, dans la mesure du possible, le temps d'échantillonnage entre deux mesures radar en prenant en compte les conditions opérationnelles de fonctionnement du radar, avant tout choisies pour les mesures de la pluie.

\section{Sélection des échos fixes}

Hubbert et al. (2009a, b) ont mis au point une mesure, la CPA (Clutter Phase Alignment), pour séparer les échos de pluie en temps réel à partir des échos de sol. La CPA est définie comme étant le rapport de l'amplitude de la somme vectorielle des membres de la série temporelle du signal radar, divisée par la somme des amplitudes du signal radar. Une approche similaire, également basée sur la phase et l'amplitude du signal, est utilisée pour définir pour chaque pixel une « stabilité à haute fréquence temporelle $\gg(\sigma)$ qui correspond à la mesure de l'inverse de la CPA. La figure 1a présente un exemple de carte de $\sigma$ obtenue durant une situation de ciel clair avec le radar de Nîmes. $\sigma$ est proche de 1 pour les échos fixes, inférieur à 0,7 pour les échos météorologiques et proche de 0 pour le bruit. Un écho mélangé (mélange d'un écho fixe et d'un écho météorologique) est présent dans l'intervalle 0,7-0,9.

Les échos fixes utilisables pour la mesure de réfractivité sont ceux dont la stabilité $\sigma$ est proche de 1 .

\section{Qualité des échos fixes}

La mesure individuelle de la pluie prend en compte une durée de signal égale au temps de résidence de l'antenne sur le pixel, voisin de $100 \mathrm{~ms}$, alors que les mesures de réfractivité utilisent des intervalles de temps beaucoup plus grands, multiples du temps qui sépare deux mesures successives (300 s). Compte tenu de cette importante différence d'échelle, la sélection des échos fixes mentionnée à la section précédente doit être complétée par une estimation du risque d'ambiguïté de mesure sur des intervalles de temps plus longs ; nous avons pour cela défini un indice de qualité $(Q I)$ égal à $2 P-1$ où $P$ est la proportion de cas où la valeur absolue de la variation de phase entre deux mesures successives est inférieure ou égale à la valeur arbitraire $\pi / 2$ qui correspond à la moitié de l'excursion totale de la phase (Besson et Parent du Châtelet, 2013). En fonction de la formulation du $Q I$, lorsque le signal radar est totalement cohérent, le $Q I$ est proche de 1 ; à l'inverse, lorsque le signal radar correspond à un bruit blanc, le $Q I$ est proche de 0 . Les valeurs négatives sont possibles, mais en pratique nous ne les observons pas. Comme on peut le constater sur la carte de $Q I$ de la figure $1 \mathrm{~b}$, les zones de fort $Q I$, également situées dans les zones déclarées échos fixes par le test du $\sigma$ (figure 1a), sont cependant moins étendues.

La distribution de $Q I$ pour les échos de sol dont le $\sigma$ est supérieur à 0,9 est représentée sur la figure 2. Il apparaît clairement sur cette figure, ainsi que pour les nombreux autres cas étudiés sur différents radars, que cette distribution est bimodale, avec un premier groupe entre -1 et 0,8 , et un pic net entre 0,9 et 1 . Ce dernier pic correspond à ceux qui sont effectivement utilisables pour les mesures de réfractivité. En effet, ces échos de sol offrent une très forte stabilité de la variation de la phase radar, puisque cette variation reste inférieure à $\pi / 2$ pendant $95 \%$ du temps. Les autres, bien qu'étant déclarés comme échos fixes par le test du $\sigma$ ne sont pas assez stables (à basse fréquence) pour servir aux mesures de réfractivité. Les pixels des autres classes ne sont pas utilisés, car ils ne présentent pas les garanties de stabilité de variation temporelle de la phase suffisante pour garantir l'absence d'ambiguité de mesure.

Le nombre de pixels utilisables pour la mesure de réfractivité (ceux présents dans la classe de $Q I[0,9 ; 1])$ est fortement dépendant du temps entre deux mesures radar (temps d'échantillonnage). Ce nombre de pixels diminue avec l'augmentation du temps d'échantillonnage (figure $2: 30 \%$ pour un intervalle de $1 \mathrm{~min}, 22 \%$ pour un intervalle de $10 \mathrm{~min})$. Il est donc nécessaire de diminuer au maximum le temps d'échantillonnage pour avoir le plus grand nombre d'échos de sol disponible pour la mesure de réfractivité.

\section{Influence \\ du temps d'échantillonnage}

Pour observer au mieux les systèmes pluvieux, les radars opérationnels sont utilisés en mode volumique : l'antenne tourne en permanence avec une vitesse azimutale généralement constante et le volume d'atmosphère est observé en faisant varier l'angle d'élévation par
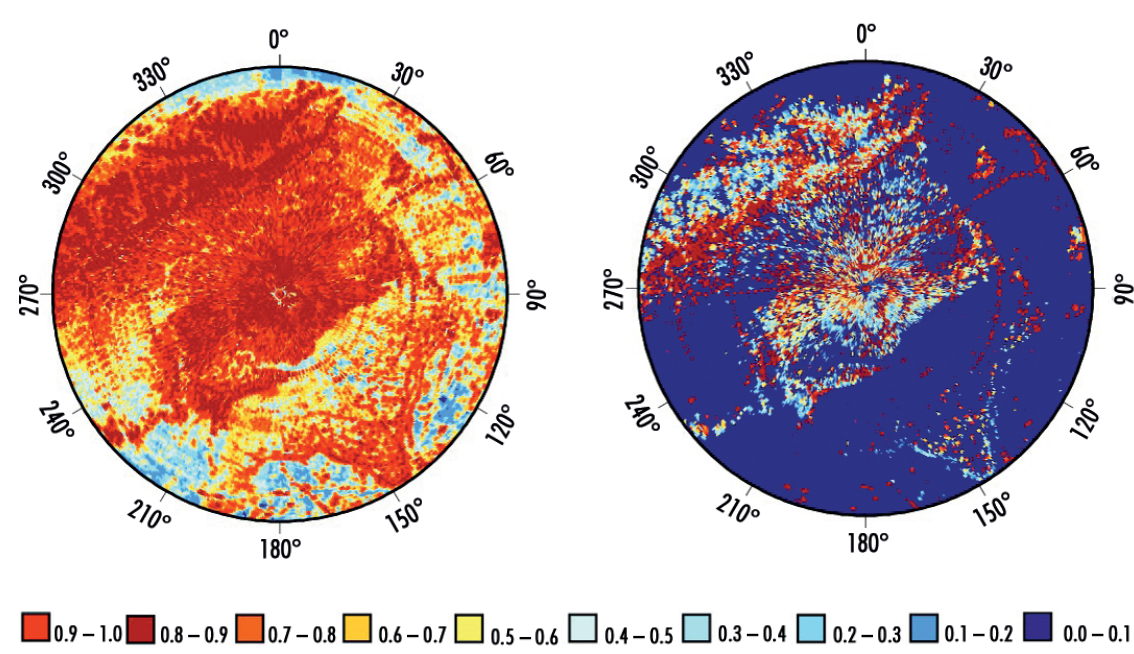

Figure 1. Tour d'antenne à l'élévation $0,6^{\circ}$ du radar de Nîmes présentant : (a) la stabilité à haute fréquence temporelle $(\sigma)$ moyennée sur 12 heures et (b) l'indice de qualité, moyenné sur 12 heures, obtenu pour les échos fixes ayant un $\sigma$ supérieur à 0,9. La distance maximale est de $35 \mathrm{~km}$.

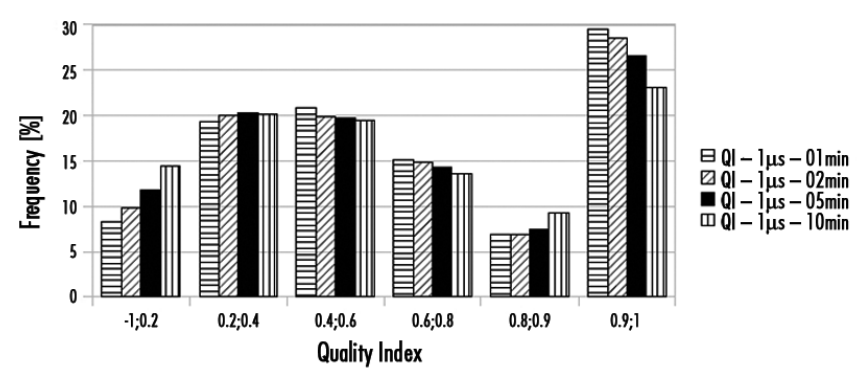

Figure 2. Histogramme de distribution de l'indice de qualité pour les 40000 pixels identi fiés comme échos de sol autour du radar de Nîmes sur les 96480 observés. Le temps d'échantillonnage varie de 1 à 10 minutes. 
pas successifs depuis les élévations les plus basses (par exemple 0,4 $4^{\circ}$ au-dessus de l'horizon) jusqu'aux plus hautes (par exemple de l'ordre de $20^{\circ}$ ). La durée de la mesure est égale au temps de résidence sur un pixel (de l'ordre de $100 \mathrm{~ms}$ ) et le temps d'échantillonnage est égal à la durée de l'exploration volumique complète (de l'ordre de $300 \mathrm{~s}$ ). Suivant les critères classiques de l'échantillonnage, ce temps $t_{\text {ech }}$ de $300 \mathrm{~s}$ permet d'échantillonner correctement un signal dont la fréquence ne dépasse pas $\pm 1 / 2 t_{\text {ech }}$, c'est-à-dire 1,6 millihertz. Nous savons que les fluctuations temporelles de la phase du signal reçu d'une cible fixe dépendent des changements atmosphériques et aussi de la complexité de la cible à travers des mécanismes que l'on ne comprend pas encore parfaitement, mais qui sont probablement liés à des interférences entre trajets multiples.

Cette dépendance est illustrée sous forme statistique sur les distributions de la figure 2 pour des valeurs du temps d'échantillonnage comprises entre 1 et $10 \mathrm{~min}$, où l'on constate que le nombre de pixels considérés comme «bons » par le critère du $Q I$ diminue sensiblement lorsque le temps d'échantillonnage augmente.

Pour pouvoir diminuer ce temps $t_{\text {éch }}$, nous avons envisagé deux solutions. La première consiste à augmenter la vitesse de rotation azimutale de l'antenne de façon à diminuer le temps total d'observation du volume d'atmosphère pour la pluie. Une telle accélération permettrait de diminuer le temps d'échantillonnage, mais aurait aussi pour effet de diminuer le temps de résidence de l'antenne sur le pixel et donc sans doute d'augmenter les erreurs sur les mesures de la pluie et de la réfractivité. La seconde consiste dans l'utilisation de plusieurs élévations successives pour réduire le temps d'échantillonnage. On cherche dans ce qui suit à évaluer les erreurs liées à ces méthodes.

\section{Erreur due}

\section{à une accélération de la rotation azimutale de l'antenne}

Besson et Parent du Châtelet (2013, résultats non montrés ici) ont mis en évidence que l'accélération de la rotation de l'antenne radar (vitesse doublée, triplée et quadruplée) a peu d'impact sur l'erreur de mesure de la réfractivité. Ce résultat confirme celui de Boudjabi
(2011), suivant lequel la phase d'un signal rétrodiffusé par une cible fixe est très cohérente et peut être correctement estimée avec un très petit nombre d'impulsions radar, voire avec une seule impulsion radar, contrairement à la mesure de la pluie dont le caractère aléatoire nécessite une certaine intégration qui justifie une résidence plus longue sur le pixel.

L'accélération de la rotation, très bénéfique pour la qualité de mesure de la réfractivité, fournirait aussi des mesures de la pluie plus fréquentes, mais éventuellement dégradées. Du point de vue de la mesure de la pluie, le bilan des avantages et inconvénients d'un tel changement des modes d'exploitation reste à faire.

\section{Utilisation d'élévations supérieures}

Pour ne pas influencer le fonctionnement opérationnel, un moyen pour diminuer artificiellement le temps entre deux mesures consiste à entrelacer des mesures effectuées à des élévations différentes. Le temps entre deux tours d'antenne à la même élévation est de 5 min. En utilisant seulement l'élévation $(A)$ la plus basse du cycle radar, le temps d'échantillonnage est donc de 5 min. En utilisant en plus de l'élévation $(A)$ l'élévation supérieur $(B)$ qui est effectuée plus tard dans le cycle radar, le temps d'échantillonnage n'est plus de 5 min, mais équivaut au temps entre l'élévation $A$ et l'élévation $B$. Il est à noter que quelle que soit l'élévation l'onde radar qui illumine l'écho de sol réalise le même trajet entre le radar et la cible. Seule l'énergie de l'onde radar diminue avec l'augmentation de l'élévation. Cette onde radar traverse donc la même atmosphère.

Besson et Parent du Châtelet (2013) établissent qu'en utilisant cette approche, c'est-à-dire en entrelaçant les séries temporelles pour les deux élévations les plus basses (correspondant à $0,4^{\circ}$ et $0,8^{\circ}$ pour le radar de Trappes), la qualité de la restitution de la réfractivité n'est pas altérée (les corrélations pour la journée du 22 février 2012 pour le radar de Trappes entre la réfractivité in situ et la réfractivité radar mesurées aux élévations $0,4^{\circ}, 0,8^{\circ}$ et l'entrelacement des deux élévations sont toujours supérieures à 0,94 ).

L'effet de la diminution du temps d'échantillonnage sur la sensibilité de la restitution de la mesure de réfractivité aux ambiguïtés de phase se traduit par une amélioration du nombre d'échos fixes utilisables pour permettre la restitution de la réfractivité (figure 3).

L'entrelacement de plusieurs élévations a pour effet positif de diminuer le temps d'échantillonnage et donc le risque d'ambiguïté. Cet effet sera d'autant plus important sur la mesure radar que les changements atmosphériques seront intenses et brutaux.

\section{Double polarisation}

Les radars les plus récents du réseau ARAMIS sont polarimétriques, c'est-àdire qu'ils peuvent simultanément émettre une onde polarisée verticalement et une autre polarisée horizontalement. L'analyse en polarisation du signal reçu permet d'obtenir des informations sur le type de particules, en particulier sur la taille des gouttes de pluie qui ont tendance à s'aplatir sous l'effet de la résistance de l'air et qui sont donc d'autant plus dissymétriques qu'elles sont grosses.

Pour savoir si cette capacité d'observation polarimétrique peut être mise à profit pour améliorer la qualité des mesures, nous avons tracé sur la figure 4 l'indice de qualité en polarisation verticale $Q I_{\mathrm{v}}$ en fonction de l'indice $Q I_{\mathrm{h}}$ en polarisation horizontale pour tous les pixels d'échos de sol du radar de Nîmes. On observe que la plupart des points sont groupés autour de la première bissectrice (région notée I sur la figure 4), ce qui revient à dire que la qualité de restitution de la réfractivité

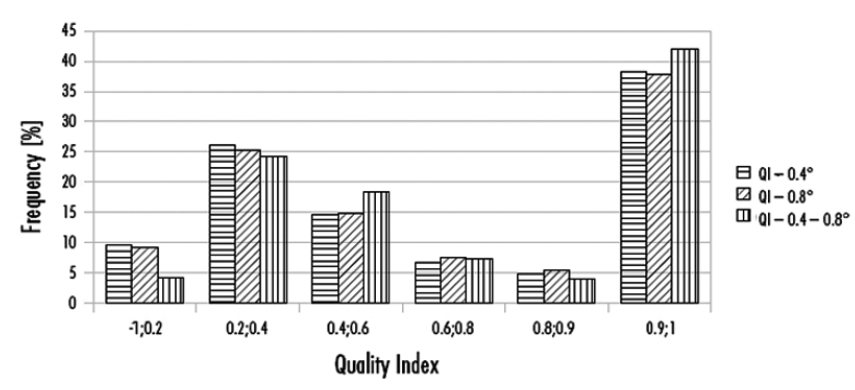

Figure 3. Histogramme de distribution de l'indice de qualité des 23000 pixels sélectionnés à partir des 96480 pixels observés autour du radar de Trappes, en fonction des élévations utilisées pour calculer l'indice $\left(0,4^{\circ}, 0,8^{\circ}\right.$ et les deux élévations $0,4^{\circ}$ et $0,8^{\circ}$. 
ne dépend pas de façon critique de la polarisation pour ces pixels. Dans ce cas, la polarimétrie ne servira qu'à augmenter le nombre de moyennes pour améliorer la précision intrinsèque de la mesure ou bien à vérifier sur le long terme que les mesures obtenues aux deux polarisations sont bien les mêmes.

La situation est différente pour les pixels (régions notées II en rouge sur la figure 4) qui ont un fort indice de qualité pour l'une des deux polarisations et un indice plus faible pour l'autre. Pour ces pixels, dont la proportion n'est pas négligeable, les chances d'avoir des variations de phase importantes qui conduisent à un sous-échantillonnage du signal sont significativement différentes pour les deux polarisations. La mesure de réfractivité radar sera donc meilleure sur l'une ou l'autre des deux polarisations. Les tests sont en cours pour déterminer une exploitation optimale de ces différences de comportement sans introduire de biais sur la mesure, mais on peut déjà dire que, lorsqu'un changement de phase important est observé sur une polarisation et pas sur l'autre, il ne s'agit pas d'une variation de réfractivité le long du trajet de l'onde puisque dans ce cas elle serait observable avec les deux polarisations. La mesure de réfractivité ne dépend pas de la polarisation de l'onde radar. Il s'agira plus probablement d'un effet non encore compris lié à la répartition spatiale de la diffusion dans le pixel, éventuellement combiné à des trajets multiples de l'onde électromagnétique.

Après que ces effets auront été compris, il est espéré que l'utilisation de la polarimétrie permette d'augmenter significativement la proportion de pixels utilisables pour la mesure de réfractivité.

\section{Comparaisons aux réfractivités simulées par Arome}

Les observations de réfractivité dans les basses couches sont potentiellement utiles pour la validation des modèles de méso-échelle et l'assimilation de données dans ces modèles. Pour que ces applications soient effectivement possibles, il faut que les modèles soient capables de représenter ce qui est observé. Afin de vérifier ce point, il est nécessaire de comparer les observations à leurs équivalents simulés par le modèle. Ceci est effectué à travers l'utilisation d'un opérateur d'observation qui agit comme un simulateur de réfractivité à partir des champs météorologiques (pression, température et humidité) pronostiqués par le modèle. Réciproquement, les comparaisons entre observations et modèle peuvent mettre à jour des observations douteuses.

De telles comparaisons ont été effectuées avec le modèle Arome dans sa configuration opérationnelle à $2,5 \mathrm{~km}$ de résolution horizontale, sur une période d'un peu plus d'un mois comportant des conditions météorologiques variées (Caumont et al., 2013). Un exemple est montré sur la figure 5 qui présente les réfractivités observées par le radar de Nîmes (à gauche) et les réfractivités simulées (à droite) le 30 mars 2011 à 0600 UTC. Les valeurs plus faibles de réfractivité à l'ouest du radar sont principalement dues à une humidité plus faible à ces endroits. On peut noter un bon accord en général entre les observations et leurs équivalents simulés par le modèle, à l'exception notable de quelques points situés au nord du radar.

Sur toute la période d'étude, la bonne adéquation entre observations et équivalents simulés par le modèle a été confir-

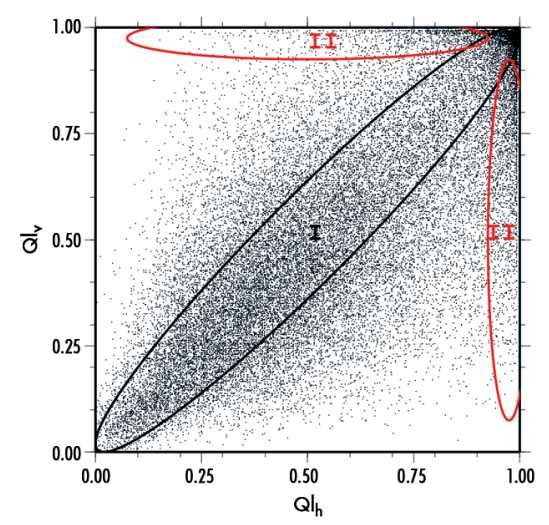

Figure 4. Nuage de points représentant pour tous les pixels radar observés l'indice de qualité calculé à parti de la variation de la phase en polarisation verticale en fonction de l'indice de qualité calculé à partir de la variation de la phase en polarisation horizontale. mée statistiquement. Cependant, des différences significatives ont été relevées pour certains événements particuliers, suggérant que l'assimilation des réfractivités mesurées par radar pouvait améliorer les états initiaux du modèle Arome dans les basses couches. Réciproquement, ces comparaisons ont permis de confirmer les disparités de qualité de la mesure de réfractivité en fonction de la nature des cibles réfléchissant les échos radar. En corollaire de ce qui avait été initialement suggéré par Fabry et al. (1997), on a ainsi vérifié que les écarts entre réfractivité observée et simulée étaient statistiquement particulièrement ténus dans les régions urbanisées qui contiennent de nombreuses cibles fixes (bâtiments, etc.) a priori de meilleure qualité pour la mesure de la réfractivité que la végétation qui peut se balancer sous l'effet du vent. Par ailleurs, une étude de sensibilité sur la modélisation du faisceau radar a permis d'établir que la mesure radar est représentative de la partie de l'atmosphère située entre le sol et une soixantaine de mètres au-dessus. Cette étude a également montré que le niveau de détail d'Arome en termes de discrétisation spatiale ne permettait pas à une description fine de la trajectoire du faisceau (par simulation explicite au sein de la grille du modèle) d'améliorer de manière visible l'adéquation entre le modèle et les observations.

\section{Application - La campagne HyMeX}

Profitant de la période d'observation spéciale de la campagne HyMeX du 5 septembre au 5 novembre 2012, dont l'objectif était d'étudier le cycle de l'eau dans la région méditerranéenne (Drobinski et al., 2013 ; Ducrocq et al., 2013), la mesure de réfractivité a été déployée sur les radars en bande $\mathrm{S}$ du réseau ARAMIS du sud de la France : les radars de Nîmes, Opoul et Bollène.

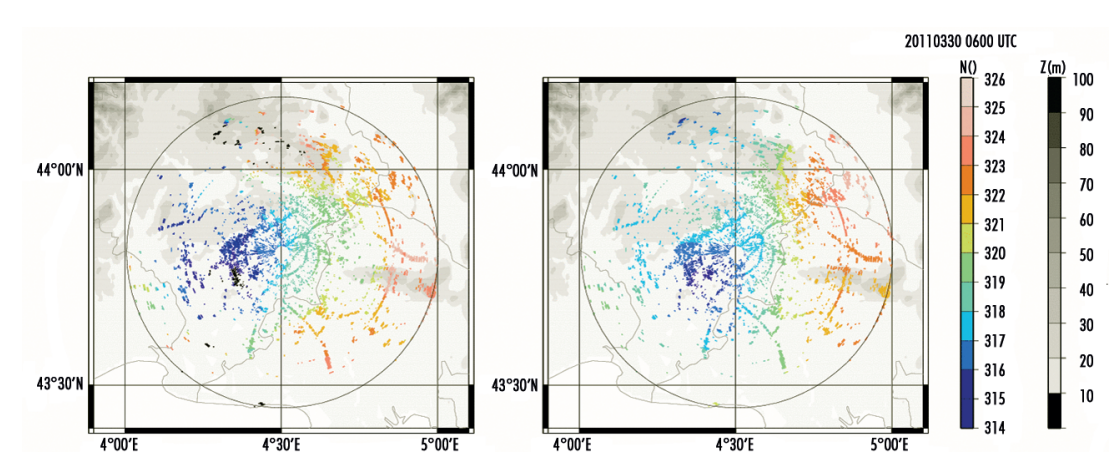

Figure 5. Réfractivité ( $N$, en plages de couleurs) vue par le radar de Nîmes le 30 mars 2011 à 0600 UTC superposée au relief au-dessus du niveau de la mer (Z, en plages de grisés) : observation (à gauche) et équivalent simulé par Arome interpolé uniquement aux points d'observation radar (à droite). Le cercle représente une portée radar de $40 \mathrm{~km}$. 

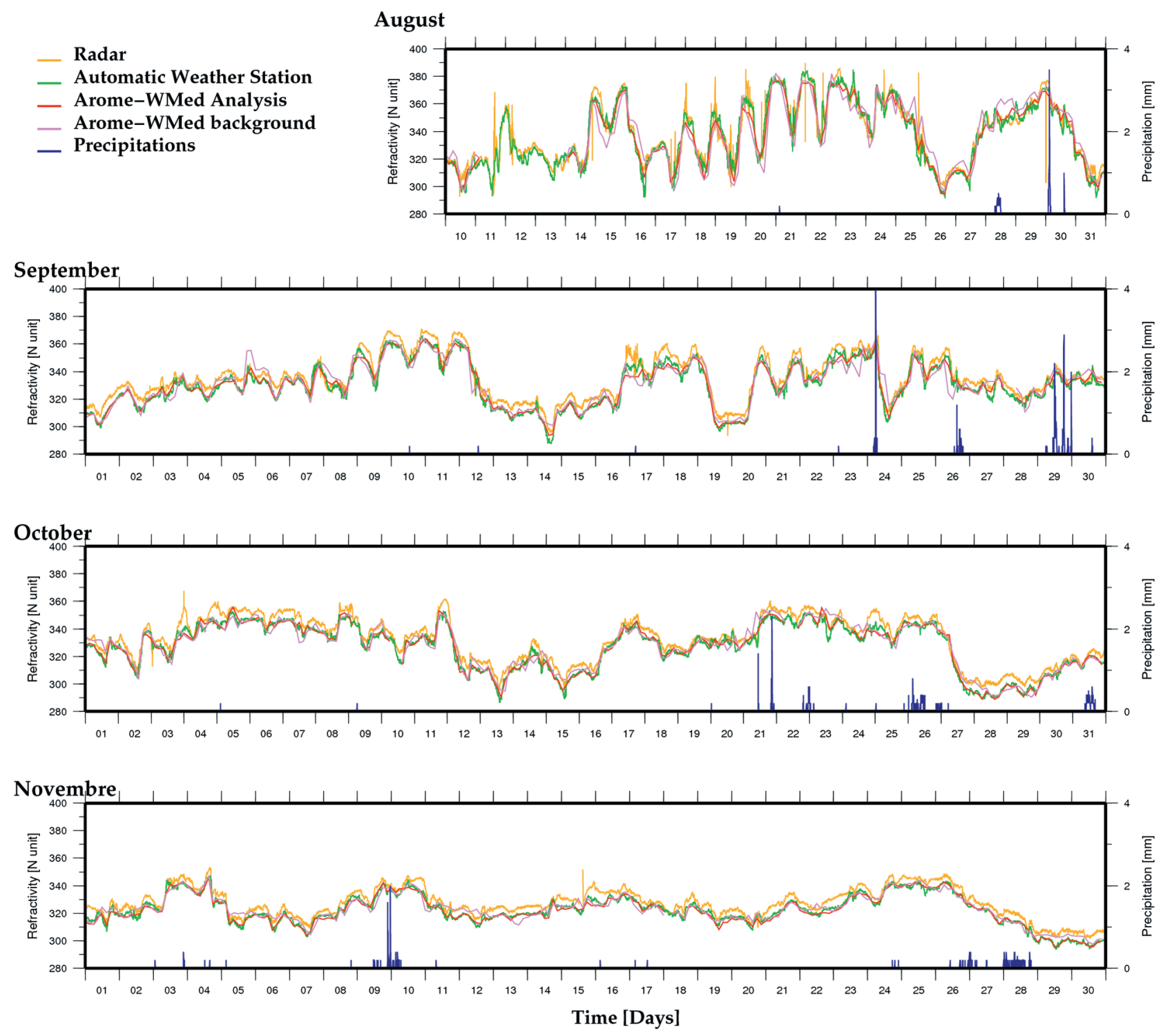

Figure 6. Comparaison des séries temporelles co-localisées de mesures et simulations de la réfractivité obtenues du 10 août au 30 novembre 2012 lors de la campagne HyMeX : réfractivité radar (orange), réfractivité in situ (vert), réfractivité issue de l'ébauche d'Arome-WMED (mauve) et réfractivité issue de l'analyse d'Arome-WMED (rouge). Les histogrammes bleus correspondent aux hauteurs d'eau mesurées par les pluviomètres lors des passages des précipitations. Un algorithme de cohérence filtre les mesures radars aberrantes, celles-ci représentant moins de 0,5\% des mesures totales effectuées pendant la campagne HyMeX. La station automatique est située à 9 km du radar.

L'algorithme de restitution de la réfractivité, développé par Fabry qui nous a gracieusement fourni le code source (Fabry et al., 1997 ; Fabry, 2004, 2006), a été implanté en temps réel pour ces trois radars après avoir été modifié et adapté aux contraintes des radars non cohérents de Météo-France (Parent du Châtelet et al., 2012).

Les objectifs étaient de valider la mesure sur une période de temps conséquente $(\approx 3$ mois et demi) englobant la période d'observation spéciale de la campagne HyMeX, d'aider à la compréhension des processus météorologiques de basses couches des systèmes observés et d'évaluer l'intérêt de la réfractivité radar pour l'assimilation de données.

\section{Validation \\ de la mesure}

La méthode de validation consiste à comparer la mesure radar de réfractivité pour des pixels co-localisés avec des mesures de référence fournies par des stations météorologiques automatiques. Pour les trois radars sélectionnés, seules sept stations sont localisées dans des zones d'échos fixes utilisables pour la réfractivité.

La comparaison (figure 6) des mesures radar (orange) et stations (vert) co-localisées au niveau de la station 30258001, située à Garons dans la région de Nîmes, permet d'illustrer le très bon accord entre les deux mesures. L'évolution temporelle, notamment le cycle diurne, est parfaitement restituée par la mesure radar. Des résultats similaires non présentés ici ont été obtenus sur l'ensemble de la période pour tous les couples pixels/stations étudiés, avec notamment des corrélations systématiquement supérieures à 0,8 . On remarque particulièrement que, même après une durée de plusieurs mois, les changements de phase des signaux par rapport à la référence du début de la période restent parfaitement exploitables pour mesurer des changements de quelques dizaines de picosecondes du retard de propagation, alors même que pendant ce temps les fréquences d'émission des radars ont varié de plusieurs centaines de kilohertz dans un sens ou dans l'autre. Cela valide parfaitement la pertinence des corrections appliquées pour prendre en compte ces variations de fréquence. 


\section{Cartographie de la réfractivité}

La mesure radar permet d'accéder à une cartographie de la mesure de la réfractivité. Ces cartes présentent potentiellement une avancée importante pour la compréhension des événements météorologiques tels que les systèmes convectifs, les fronts, les brises...

Par exemple, l'étude des cartes de réfractivité produites par le radar de Nîmes lors de la journée du 26 septembre 2012 permet de suivre l'évolution des masses d'air en utilisant le code source de F. Fabry (figure 7).

Ces cartes permettent d'identifier qu'une masse d'air sèche et chaude, venant de l'ouest, va s'installer sur l'ensemble du territoire et remplacer la masse d'air plus humide/froide qui était initialement présente.

Lapport de ces cartes de réfractivité est ainsi indéniable pour la compréhension des processus de très basses couches.

\section{Comparaison entre la réfractivité radar et la réfractivité simulée par Arome}

La comparaison sur trois mois et demi entre le pixel radar co-localisé à la station météorologique a été étendue aux données simulées à partir des ébauches et des analyses tri-horaires d'AromeWMED, la version d'Arome spécialement déployée pendant la campagne HyMeX pour guider le déploiement des plates-formes instrumentales (avions, ballons, etc.).
Les résultats préliminaires (figure 6) montrent que les quatre jeux de données sont en très bon accord $(R>0,78)$. La réfractivité radar et la réfractivité de la station automatique sont cependant plus proches de la réfractivité issue de l'analyse (rouge) que de celle provenant de l'ébauche (mauve), confirmant l'apport de l'assimilation des données mesurées par les stations automatiques dans le modèle ${ }^{1}$.

Cette étude demande à être poursuivie et étendue aux pixels non co-localisés avec les stations météorologiques pour étudier les différences entre la mesure radar et le modèle, et déterminer dans quelles conditions l'assimilation des données de réfractivité mesurées par radar est susceptible d'avoir un effet bénéfique maximal.

\section{Perspectives}

La mesure de la réfractivité radar est une avancée importante dans l'étude des paramètres météorologiques dans les très basses couches de l'atmosphère. Son adaptation aux radars non cohérents permet maintenant d'étendre son utilisation à l'ensemble des réseaux opérationnels des organismes météorologiques.

Nous avons constaté que certains échos de sol sont exploitables pour les mesures, alors que d'autres ne le sont pas. L'utilisation d'un indice de qualité permet de quantifier cette capacité et donc d'améliorer la fiabilité de la mesure. L'origine physique de ces erreurs, qui n'est pas encore comprise, est certainement une des clés de l'amélioration de la mesure dans un futur proche.
Avant toute tentative d'utilisation opérationnelle, les travaux de validation effectués devront être complétés et consolidés afin de permettre de fournir aux utilisateurs une mesure de réfractivité de très haute qualité. Ces améliorations passeront principalement par une meilleure compréhension de la physique à l'origine des fluctuations de phase des échos de sol, suivant que la cible est ponctuelle et isolée ou bien répartie, ainsi que par des études sur l'impact de la turbulence atmosphérique sur la propagation de l'onde radar.

Un programme de travail, défini dans ce sens, est en cours en association entre des partenaires du CNRM, du Latmos et de certains centres météorologiques régionaux, afin d'étudier le potentiel de la réfractivité pour l'assimilation dans les modèles et pour l'aide à la prévision
1. L'ébauche désigne une prévision récente du modèle qui est combinée avec les observations (on dit que ces dernières sont assimilées) afin d'obtenir la meilleure estimation possible de l'état de l'atmosphère. Cette estimation est appelée analyse et sert de conditions initiales pour la prévision suivante. En ce qui concerne Arome, des analyses sont produites toutes les trois heures à partir d'ébauches qui sont des prévisions à trois heures d'échéance initialisées à partir de l'analyse précédente.
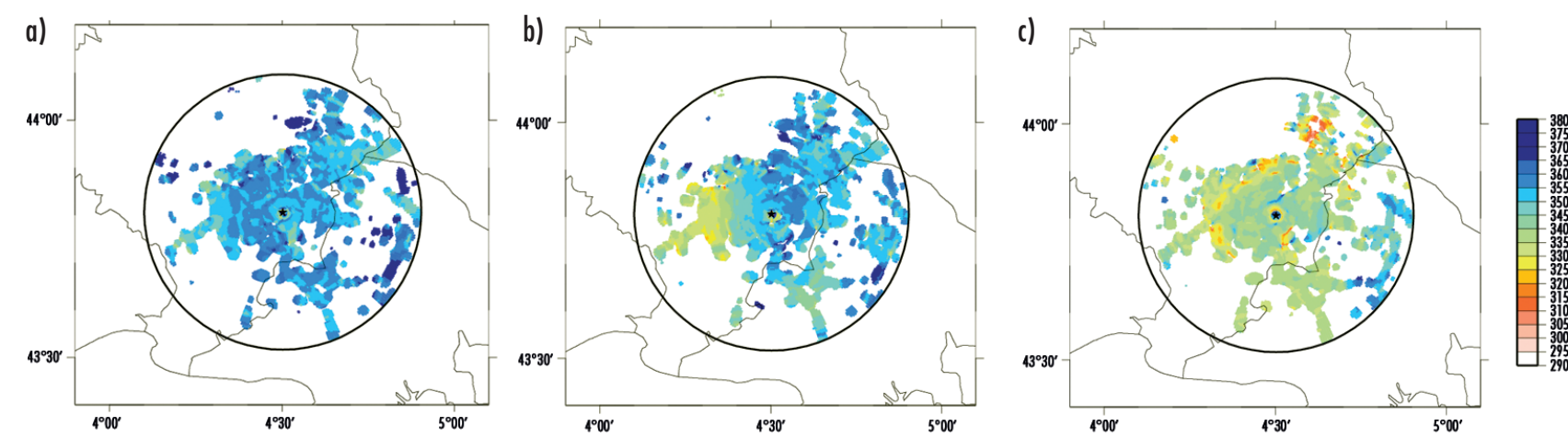

Figure 7. Cartes de réfractivité réalisées par le radar de Nîmes le 26 septembre 2012 à 9 h30 (a), 11 h00 (b) et 13 h55 (c) pendant la campagne HyMeX. La masse d'air de forte réfractivité présente à $9 \mathrm{~h} 30$ est remplacée à $13 \mathrm{~h} 55$ par une masse d'air de plus faible réfractivité provenant de l'ouest. Cette évolution atmosphérique de très basses couches correspond à un assèchement et/ou un réchauffement relatif. 


\section{Bibliographie}

Bean B.R. et E.J. Dutton, 1968 : Radio Meteorology. National Bureau of Standards Monogr., No. 92, National Bureau of Standards, 435 p.

Besson L. et J. Parent du Châtelet, 2013 : Solutions for improving the radar refractivity measurement by taking operational constraints into account. J. Atmos. Oceanic Technol., 30, 1730-1742.

Besson L., C. Boudjabi, 0. Caumont et J. Parent du Châtelet, 2012 : Links between weather phenomena and characteristics of refractivity measured by precipitation radar. Bound.-Layer Meteor., 143, 77-95.

Bodine D., P.L. Heinselman, B.L. Cheong, R.D. Palmer et D. Michaud, 2010 : A case study on the impact of moisture variability on convection initiation using radar refractivity retrievals. J. Appl. Meteor. Climatol., 49, 1766-1778.

Boudjabi C., 2011 : Validation de la mesure de réfractivité avec un radar en bande C équipé d'un émetteur à magnétron. Doctorat de l'Université Paul Sabatier, Toulouse, soutenu le 24 février 2011.

Caumont 0., A. Foray, L. Besson et J. Parent du Châtelet, 2013 : An observation operator for radar reflectivity change: comparison of observation and convective-scale simulations. Bound.-Layer Meteor., 148, 379-397.

Dabas A. et C. Flamant, 2002 : Les instruments de télédétection au sol. La Météorologie, 39, 76-82.

Demoz B., C. Flamant, T. Weckwerth, D. Whiteman, K. Evans, F. Fabry, P. Di Girolamo, D. Miller, B. Geerts, W. Brown, G. Schwemmer, B. Gentry, W. Feltz et Z. Wang, 2006 : The dryline on 22 May 2002 during IHOP 2002: Convective-scale measurements at the profiling site. Mon. Wea. Rev., 134, 294-310

Doerflinger E., 2001 : Les applications météorologiques du système de positionnement satellitaire GPS. La Météorologie, 34, 21-37.

Drobinski P., V. Ducrocq, P. Arbogast, C. Basdevant, S. Bastin, L. Beguery, S. Belamari, K. Béranger, O. Bock, M.-N. Bouin, B. Boudevillain, O. Bousquet, C. Bouvier, I. Braud, J.-C. Calvet, C. Champollion, A. Chanzy, M. Claude, P. Cocquerez, L. Coppola, S. Coquillat, D. Courault, E. Defer, J. Delanoë, G. Delrieu, A. Doerenbecher, N. Dorfliger, M. Estèves, C. Estournel, C. Flamant, N. Fourrie, 0. Garrouste, E. Gaume, H. Giordani, L. Gomes, G. Roberts, H. Jourde, L. Labatut, D. Lambert, J. Le Coz, W. Ludwig, C. Lutoff, J.-F. Mahfouf, E. Martin, L. Mastrorillo, N. Mathys, Y. Michel, M. Nuret, F. Orain, S. Perez, C. Prigent, O. Radakovitch, K. Ramage, E. Richard, O. Roussot, I. Ruin, F. Saiid, A. Schwarzenboeck, K. Sellegri, S. Somot, I. Taupier-Letage, J.-L. Roujean, P. Testor, J. Van Baelen et J.-P. Vandervaere, 2013 : HyMeX, le cycle de l'eau méditerranéen à la loupe. La Météorologie, 80, 23-36.

Ducrocq V., S Belamari, B. boudevillain, O. Bousquet, P. Cocquerez, A. Doerenbecher, P. Drobinski, C. Flamant, L. Labatut, D. Lambert, M. Nuret, E. Richard, 0. Roussot, P. Testor, P. Arbogast, P. A. Ayral, J. Van Baelen, C. Basdevant, J.-L. Boichard, D. Bourras, C. Bouvier, M.-N. Bouin, O. Bock, I. Braud, C. Champollion, L. Coppola, S. Coquillat, E. Defer, J. Delanoë, G. Delrieu, P. Durand, C. Estournel, N. Fourié, O. Garrouste, H. Giordani, J. Le Coz, Y. Michel, O. Nuissier, G. Roberts, F. Said, A. Schwarzenboeck, K. Sellegri, I. Taupier-Letage et J. P. Vandervaere, 2013 : HyMeX, les campagnes de mesure : focus sur les événements extrêmes en Méditerranée. La Météorologie, 80, 3747.

Fabry F., 2004 : Meteorological value of ground target measurements by radar. J. Atmos. Oceanic Technol., 21, 560-573.

Fabry F., 2006 : The spatial variability of moisture in the boundary layer and its effect on convection initiation: Project-long characterization. Mon. Wea. Rev., 134, 79-91.

Fabry F., C. Frush, I. Zawadzki et A. Kilanbi, 1997 : On the extraction of near-surface index of refraction using radar phase measurements from ground targets. J. Atmos. Oceanic Technol., 14, 978-987.

Gossard E.E., D.E. Wolfe, K.P. Moran, R.A. Paulus, K.D. Anderson et L.T. Rogers, 1998 : Measurement of clear-air gradients and turbulence properties with radar wind profilers. J. Atmos. Oceanic Technol., 15, 321-342.

Heinselman P.L., B.L. Cheong, R.D. Palmer, D. Bodine et K. Hondl, 2009 : Radar refractivity retrievals in Oklahoma: Insights into operational benefits and limitations. Wea. Forecasting, 24, 1345-1361.

Hubbert J.-C., M. Dixon et S.M. Ellis, 2009a : Weather radar ground clutter. Part II: Real-time identification and filtering. J. Atmos. Oceanic Technol., 26, 1181-1197.

Hubbert J.-C., M. Dixon, S.M. Ellis et G. Meymari, 2009b : Weather radar ground clutter. Part I: Identification, modelling and simulation. J. Atmos. Oceanic Technol., 26, 1165-1180.

Montmerle T., A. Caya et I. Zawadzki, 2002 : Short-term numerical forecasting of a shallow storms complex using bistatic and single-doppler radar data. Wea. Forecasting, $17,1211-1225$

Parent du Châtelet J., C. Boudjabi, L. Besson et 0. Caumont, 2012 : Errors caused by long-term drifts of magnetron frequencies for refractivity measurement with a radar: Theoretical formulation and initial validation. J. Atmos. Oceanic Technol., 29, 1428-1434.

Roberts R.D., F. Fabry, P.C. Kennedy, E. Nelson, J.W. Wilson, N. Rehak, J. Fritz, V. Chandrasekar, J. Braun, J. Sun, S. Ellis, S. Reising, T. Crum, L. Mooney, R. Palmer, T. Weckwerth, et S. Padmanabhan, 2008 : REFRACTT 2006. Real-time retrieval of high-resolution, low-level moisture fields from operational NEXRAD and research radars. Bull. Amer. Meteor. Soc., 89, 1535-1548.

Smith E.K.J. et S. Weintraub, 1953 : The constants in the equation for atmospheric refractive index at radio frequencies. Proceedings of the Institute of Radio Engineers, 41 , 1035-1037.

Stankov B.B., E.E. Gossard, B.L. Weber, R.J. Lataitis, A.B. White, D.E. Wolfe, D.C. Welsh et R.G. Strauch, 2003 : Humidity gradient profiles from wind profiling radars using the NOAA/ETL advanced signal processing system (SPS). J. Atmos. Oceanic Technol., 20, 3-22.

Sun J., 2005 : Convective-scale assimilation of radar data: Progress and challenges. Quart. J. Roy. Meteor. Soc., 131, 3439-3463.

Wakimoto R.M. et H.V. Murphey, 2010 : Frontal and radar refractivity analyses of the dryline on 11 June 2002 during IHOP. Mon. Wea. Rev., 138, 228-240.

Weckwerth T.M., C.R. Pettet, F. Fabry, S. Park, M.A. Lemone et J.W. Wilson, 2005 : Radar refractivity retrieval: Validation and application to short-term forecasting. J. Appl. Meteorol., 44, 285-300. 